\title{
Units of Measurements and Ming and Qing Reign Names and Dates
}

UNITS OF MEASUREMENTS
I $m u=1 / 6$ acre
I catty $=\mathrm{I} .33 \mathrm{lb}$.
I qing $=100 m u=16.7$ acres
I tael $=\mathbf{I} .33 \mathrm{Oz}$. of silver

MING AND QING REIGN NAMES AND DATES

Ming

\begin{tabular}{|c|c|c|c|}
\hline Hongwu & I368-98 & Hongzhi & I488-I 505 \\
\hline Jianwen & I399-I4O2 & Zhengde & I 506-2I \\
\hline Yongle & $1403-24$ & Jiajing & $1522-66$ \\
\hline Hongxi & 1425 & Longqing & $1567-72$ \\
\hline Xuande & $1426-35$ & Wanli & I $573-I 620$ \\
\hline Zhengtong & $1436-49$ & Taichang & 1620 \\
\hline Jingtai & I450-57 & Tianqi & $162 I-27$ \\
\hline Tianshun & I $457-64$ & Chongzhen & I $628-44$ \\
\hline Chenghua & $1465-87$ & & \\
\hline
\end{tabular}

Qing

$\begin{array}{llll}\text { Shunzhi } & \text { I644-6I } & \text { Daoguang } & \text { I82I-50 } \\ \text { Kangxi } & \text { I662-I722 } & \text { Xianfeng } & \text { I85I-6I } \\ \text { Yongzheng } & \text { I723-35 } & \text { Tongzhi } & \text { I862-74 } \\ \text { Qianlong } & \text { I736-95 } & \text { GuangXu } & \text { I875-I908 } \\ \text { Jiaqing } & \text { I796-I820 } & \text { Xuantong } & \text { I909-II }\end{array}$

